DOI: 10.17516/1997-1397-2021-14-4-483-491

УДК 517.9

\title{
On the Solvability of the Identification Problem for a Source Function in a Quasilinear Parabolic System of Equations in Bounded and Unbounded Domains
}

\author{
Vera G. Kopylova* \\ Igor V. Frolenkov ${ }^{\dagger}$ \\ Siberian Federal University \\ Krasnoyarsk, Russian Federation
}

Received 10.01.2021, received in revised form 05.04.2021, accepted 20.05.2021

\begin{abstract}
The paper considers the problem of identification for a source function in one of two equations of parabolic quasilinear system. The case of Cauchy data in an unbounded domain and the case of boundary conditions of the first kind in a rectangular domain are considered. The question of the existence and uniqueness of the solution is studied. The proof uses a differential level splitting method known as the weak approximation method. The solution is obtained on a small time interval in the class of sufficiently smooth bounded functions.
\end{abstract}

Keywords: inverse problem, quasilinear equations system, source function determination, weak approximation method, small parameter.

Citation: V.G. Kopylova, I.V. Frolenkov, On the Solvability of the Identification Problem for a Source Function in a Quasilinear Parabolic System of Equations in Bounded and Unbounded Domains, J. Sib. Fed. Univ. Math. Phys., 2021, 14(4), 483-491. DOI: 10.17516/1997-1397-2021-14-4-483-491.

This work is dedicated to the memory of our teacher, Doctor of Physical and Mathematical Sciences, Yuri Yakovlevich Belov, who was a recognized and famous specialist in inverse problems of mathematical physics. A number of his latest papers were devoted to the study of solvability of inverse problems for parabolic-elliptic semi-evolutionary systems of differential equations. To study the existence of solutions to such inverse problems, Yu. Belov suggested using the wellknown $\varepsilon$-approximation method. The essence of the method for a system of parabolic and elliptic equations, for example, is to replace the elliptic equation with a parabolic one, which contains a small parameter $\varepsilon$ at the time derivative. The method was proposed by N. N. Yanenko who suggested replacing the Navier-Stokes equations of a viscous incompressible fluid with equations of the Cauchy-Kovalevskaya type with a small parameter $\varepsilon$. Thus, as $\varepsilon$ approaches to zero, the approximating equations become the original ones.

The main part of the mentioned works by Yu. Ya Belov is devoted to the study of linear parabolic-elliptic systems. The case when the unknown component of the source vector function is in an equation that does not contain a small parameter, under the first and second boundary conditions were studied in [1,2]. In [3], a one-dimensional system is considered, in which the unknown component of the source vector function is in the equation containing the $\varepsilon$ parameter. In the case when the components of the vector function are unknown in each equation of the system, the Cauchy problem and the first boundary value problem are investigated in [4].

\footnotetext{
*kopylova.vera@mail.ru

†igor@frolenkov.ru https://orcid.org/0000-0001-7223-8112

(c) Siberian Federal University. All rights reserved
} 
The research scheme in these works, as a rule, assumes that we first have to investigate the solvability of the auxiliary approximating problem (in an unbounded domain with Cauchy data and / or in a bounded domain with boundary conditions of the first or second kind), since these problems are nonclassical problems for loaded equations, and investigate the necessary properties of solutions that obviously depend on the parameter $\varepsilon$. And then the second step is to obtain estimates that will guarantee the convergence of the sequence of solutions of the approximating problem to the solution of the original problem as $\varepsilon$ approaches zero.

In this paper, a quasilinear system of two parabolic equations with one unknown coefficient of the source function is considered. The question of a solution existence to this problem is studied. This is a model problem in which the authors set the goal of working out the splitting algorithm and obtaining a priori estimates for quasilinear systems, which is much more complicated than in the linear case. It is also important to note that the system under consideration contains a small fixed parameter $\varepsilon>0$, which does not affect the study of the question of a solution existence, but allows using this system subsequently as an approximating model for the problem of identifying the source function in a quasilinear parabolic-elliptic system.

\section{Formulation of the problem and reduction it to the direct problem}

Consider in the strip $G_{[0, T]}=\left\{(t, x) \mid 0 \leqslant t \leqslant T, x \in E_{1}\right\}$ the problem of determining real-valued functions $(u(t, x), v(t, x), r(t))$, satisfying the system of equations

$$
\left\{\begin{array}{l}
u_{t}(t, x)+a_{11}(t) u(t, x)+a_{12}(t) v(t, x)=\mu_{1} u_{x x}(t, x)+v(t, x) u_{x}(t, x)+r(t) f(t, x), \\
\varepsilon v_{t}(t, x)+a_{21}(t) u(t, x)+a_{22}(t) v(t, x)=\mu_{2} v_{x x}(t, x)+u(t, x) v_{x}(t, x)+g(t, x),
\end{array}\right.
$$

where $\varepsilon \in(0,1]$ is a const, with initial conditions

$$
u(0, x)=u_{0}(x), \quad v(0, x)=v_{0}(x),
$$

and the over determination condition

$$
u\left(t, x^{0}\right)=\varphi(t)
$$

where $\varphi(t)$ is a given function on $[0, T], 0 \leqslant t \leqslant T, x^{0}$ is a fixed point.

System (1), for example, can be an approximation of the parabolic-elliptic system of equations

$$
\left\{\begin{aligned}
\tilde{u}_{t}(t, x)+a_{11}(t) \tilde{u}(t, x)+a_{12}(t) \tilde{v}(t, x) & =\mu_{1} \tilde{u}_{x x}(t, x)+\tilde{v}(t, x) \tilde{u}_{x}(t, x)+\tilde{r}(t) f(t, x), \\
a_{21}(t) \tilde{u}(t, x)+a_{22}(t) \tilde{v}(t, x) & =\mu_{2} \tilde{v}_{x x}(t, x)+\tilde{u}(t, x) \tilde{v}_{x}(t, x)+g(t, x) .
\end{aligned}\right.
$$

Note that the study of the behavior of the solution when $\varepsilon$ approaches zero is beyond the scope of this study, and in our work $\varepsilon$ is a nonnegative fixed constant.

Let the functions $a_{i j}(t), i, j=1,2$, be defined on $[0, T]$ and let the functions $f(t, x), g(t, x)$ be defined on $G_{[0, T]}$. Let $\mu_{1}, \mu_{2}>0$ be given constants.

Let the relationship

$$
\left|f\left(t, x^{0}\right)\right| \geqslant \delta>0, \quad t \in[0, T] \quad(\delta \text { is a const })
$$

hold. 
Assume that the following consistency condition is fulfilled

$$
u_{0}\left(x^{0}\right)=\varphi(0) .
$$

Reduce the inverse problem (1)-(2) to an auxiliary direct problem. In system (1) we set $x=x^{0}$ :

$$
\left\{\begin{array}{l}
\varphi^{\prime}(t)+a_{11}(t) \varphi(t)+a_{12}(t) v\left(t, x^{0}\right)=\mu_{1} u_{x x}\left(t, x^{0}\right)+v\left(t, x_{0}\right) u_{x}\left(t, x^{0}\right)+r(t) f\left(t, x^{0}\right), \\
\varepsilon v_{t}\left(t, x^{0}\right)+a_{21}(t) \varphi(t)+a_{22}(t) v\left(t, x^{0}\right)=\mu_{2} v_{x x}\left(t, x^{0}\right)+\varphi(t) v_{x}\left(t, x^{0}\right)+g\left(t, x^{0}\right) .
\end{array}\right.
$$

From (6) we obtain

$$
r(t)=\frac{\psi(t)+a_{12} v\left(t, x^{0}\right)-\mu_{1} u_{x x}\left(t, x^{0}\right)-v\left(t, x_{0}\right) u_{x}\left(t, x^{0}\right)}{f\left(t, x^{0}\right)} .
$$

where $\psi(t)=\varphi^{\prime}(t)+a_{11}(t) \varphi(t)$ is known.

Substituting expression for $r(t)$ in (1) we obtain the following direct problem:

$$
\left\{\begin{aligned}
u_{t}(t, x)+a_{11}(t) u(t, x)+a_{12}(t) v(t, x) & =\mu_{1} u_{x x}(t, x)+v(t, x) u_{x}(t, x)+ \\
+\frac{\psi(t)+a_{12}(t) v\left(t, x^{0}\right)-\mu_{1} u_{x x}\left(t, x^{0}\right)-v\left(t, x^{0}\right) u_{x}\left(t, x^{0}\right)}{f\left(t, x^{0}\right)} f(t, x) & \\
\varepsilon v_{t}(t, x)+a_{21}(t) u(t, x)+a_{22}(t) v(t, x) & =\mu_{2} v_{x x}(t, x)+u(t, x) v_{x}(t, x)+g(t, x), \\
u(0, x) & =u_{0}(x), \\
v(0, x) & =v_{0}(x) .
\end{aligned}\right.
$$

\section{Proof of solvability of the problem (1)-(3)}

To prove the existence of a solution to the auxiliary problem (1)-(3), we use the weak approximation method $[5,6]$. We split the problem (8)-(10) and linearize it by shifting in time by $\frac{\tau}{4}$.

$$
\begin{gathered}
\left\{\begin{array}{l}
u_{t}^{\tau}(t, x)=4 \mu_{1} u_{x x}^{\tau}(t, x), \\
\varepsilon v_{t}^{\tau}(t, x)=4 \mu_{2} v_{x x}^{\tau}(t, x), \quad j \tau<t \leqslant\left(j+\frac{1}{4}\right) \tau,
\end{array}\right. \\
\left\{\begin{array}{l}
u_{t}^{\tau}(t, x)+4 a_{11}(t) u^{\tau}(t, x)=0, \\
\varepsilon v_{t}^{\tau}(t, x)+4 a_{22}(t) v^{\tau}(t, x)=0,
\end{array} \quad\left(j+\frac{1}{4}\right) \tau<t \leqslant\left(j+\frac{1}{2}\right) \tau,\right. \\
\left\{\begin{array}{l}
u_{t}^{\tau}(t, x)=4 v^{\tau}\left(t-\frac{\tau}{4}, x\right) u_{x}^{\tau}(t, x), \\
\varepsilon v_{t}^{\tau}(t, x)=4 u^{\tau}\left(t-\frac{\tau}{4}, x\right) v_{x}^{\tau}(t, x),
\end{array} \quad\left(j+\frac{1}{2}\right) \tau<t \leqslant\left(j+\frac{3}{4}\right) \tau,\right. \\
=4 \frac{\psi(t)+a_{12}(t) v^{\tau}\left(t-\frac{\tau}{4}, x^{0}\right)-\mu_{1} u_{x x}^{\tau}\left(t-\frac{\tau}{4}, x^{0}\right)-v^{\tau}\left(t-\frac{\tau}{4}, x^{0}\right) u_{x}^{\tau}\left(t-\frac{\tau}{4}, x^{0}\right)}{f\left(t, x^{0}\right)} f(t, x), \\
\varepsilon v_{t}^{\tau}(t, x)+4 a_{21}(t) u^{\tau}\left(t-\frac{\tau}{4}, x\right)=4 g(t, x), \quad\left(j+\frac{3}{4}\right) \tau<t \leqslant(j+1) \tau,
\end{gathered}
$$




$$
\begin{aligned}
& \left.u^{\tau}(t, x)\right|_{t \leqslant 0}=u_{0}(x), \\
& \left.v^{\tau}(t, x)\right|_{t \leqslant 0}=v_{0}(x) .
\end{aligned}
$$

Here $j=0,1, \ldots, N-1 ; \tau N=T$.

Concerning the input data, assume that they are sufficiently smooth, have all continuous derivatives occurring in the next lower relations of (17)-(19) and satisfy them:

$$
\begin{gathered}
\left|a_{i j}(t)\right| \leqslant C, \quad i=1,2, \quad j=1,2, \\
\left|\frac{\partial^{k}}{\partial x^{k}} f(t, x)\right|+\left|\frac{\partial^{k}}{\partial x^{k}} F(t, x)\right|+\left|\frac{d^{k}}{d x^{k}} u_{0}(x)\right|+\left|\frac{d^{k}}{d x^{k}} v_{0}(x)\right| \leqslant C, \quad k=0, \ldots, p+6, \\
|\varphi(t)|+\left|\varphi^{\prime}(t)\right| \leqslant C, \quad(t, x) \in G_{[0, T]} .
\end{gathered}
$$

Below, for convenience, we consider some proofs assuming that the constant $C$ is greater than 1 and that the constant $p \geqslant 6$ is an even number.

For the solution $u^{\tau}(t, x), v^{\tau}(t, x)$ of the split linearized problem (9)-(12) are obtained a priori estimates uniform in $\tau$ for $j=0,1, \ldots, p+1, k=0,1, \ldots, p,(t, x) \in G_{[0, T]}$

$$
\left|\frac{\partial^{p+4}}{\partial x^{p+4}} u_{t}^{\tau}(t, x)\right|+\left|\frac{\partial^{p+4}}{\partial x^{p+4}} v_{t}^{\tau}(t, x)\right| \leqslant C, \quad(t, x) \in G_{\left[0, t^{*}\right]},
$$

where $t^{*}$ does not depend on $\tau$ and depends on $\varepsilon$.

By virtue of the (20), the theorem of Arzela [7] and the convergence theorem of the weak approximation method [6], it follows that the limit functions $u(t, x), v(t, x)$ for $\tau \rightarrow 0$ are a solution to the direct problem (8)-(10), and $u(t, x), v(t, x)$ and $r(t, x)$ defined by relation (7) are solutions of problem (1), (2).

The uniqueness of the found solution is proved in a standard way, by obtaining estimates showing that the difference of two possible solutions in $G_{\left[0, t^{*}\right]}$ is equal to zero.

The following theorem gives sufficient conditions for the existence and uniqueness of a solution.

Theorem 2.1. Let the conditions (4), (5), (17)-(19) hold. Then there exists a unique solution $u(t, x), v(t, x), r(t)$ of problem (1)-(3) in the class

$$
\begin{aligned}
Z\left(t^{*}\right)=\left\{u(t, x), v(t, x), r(t) \mid u(t, x) \in C_{t, x}^{1, p+4}\left(G_{\left[0, t^{*}\right]}\right), v(t, x) \in C_{t, x}^{1, p+4}\left(G_{\left[0, t^{*}\right]}\right),\right. & \\
& \left.r(t) \in C\left(\left[0, t^{*}\right]\right)\right\},
\end{aligned}
$$

and the following relations hold

$$
\begin{aligned}
\sum_{k=0}^{p+4}\left(\left|\frac{\partial^{k}}{\partial x^{k}} u(t, x)\right|+\left|\frac{\partial^{k}}{\partial x^{k}} v(t, x)\right|\right)+\left.|| r(t)\right|_{C^{1}\left[0, t^{*}\right]}+\left|\frac{\partial}{\partial t} u(t, x)\right|+\left|\frac{\partial}{\partial t} v(t, x)\right| & \leqslant C(\varepsilon), \\
(t, x) & \in G_{\left[0, t^{*}\right]} .
\end{aligned}
$$

where

$$
C_{t, x}^{1, p+4}\left(G_{\left[0, t^{*}\right]}\right)=\left\{u(t, x) \mid u_{t} \in C\left(G_{\left[0, t^{*}\right]}\right), \frac{\partial^{k}}{\partial x^{k}} u \in C\left(G_{\left[0, t^{*}\right]}\right), k=0, \ldots, p+4\right\} .
$$

Obviously, the solution depends on the constant $\varepsilon$, just as the constant $C(\varepsilon)$ depends on $\varepsilon$ and the input data. 


\section{Periodicity}

In the domain $Q_{t^{*}}=\left\{(t, x) \mid 0<t<t^{*}, 0<x<l\right\}$ consider the boundary value problem

$$
\left\{\begin{array}{l}
u_{t}(t, x)+a_{11}(t) u(t, x)+a_{12}(t) v(t, x)=\mu_{1} u_{x x}(t, x)+v(t, x) u_{x}(t, x)+r(t) f(t, x), \\
\varepsilon v_{t}(t, x)+a_{21}(t) u(t, x)+a_{22}(t) v(t, x)=\mu_{2} v_{x x}(t, x)+u(t, x) v_{x}(t, x)+g(t, x),
\end{array}\right.
$$

$\varepsilon$ is a const, $\varepsilon \in(0,1]$,

$$
\begin{gathered}
u(0, x)=u_{0}(x), \quad x \in[0, l], \\
v(0, x)=v_{0}(x), \quad x \in[0, l], \\
u(t, 0)=u(t, l)=v(t, 0)=v(t, l)=0, \quad t \in\left[0, t^{*}\right], \\
u\left(t, x^{0}\right)=\varphi(t), \quad 0<x_{0}<l, \\
u_{0}\left(x^{0}\right)=\varphi(0) .
\end{gathered}
$$

Let us extend the functions $u_{0}(x), v_{0}(x), f(t, x), g(t, x)$ to the segment $[-l, l]$ :

$$
\begin{aligned}
& u_{0}(x)=-u_{0}(-x), \quad \text { for }-l \leqslant x<0, \\
& v_{0}(x)=-v_{0}(-x), \text { for }-l \leqslant x<0 .
\end{aligned}
$$

Then we continue the functions from $[-l, l]$ to $\Re$ in a periodic manner.

Extend the functions $f(t, x)$ and $g(t, x)$ from $\left[0, t^{*}\right] \times[0, l]$ to $\left[0, t^{*}\right] \times \Re$ to periodic and odd in $x$ functions.

Note that the functions $u_{0}(x), v_{0}(x), f(t, x), g(t, x)$, according to the construction method, satisfy the conditions:

$$
\begin{array}{cl}
u_{0}(-x)=-u_{0}(x), & u_{0}(l-x)=-u_{0}(l+x), \\
v_{0}(-x)=-v_{0}(x), & v_{0}(l-x)=-v_{0}(l+x), \\
f(t,-x)=-f(t, x), & f(t, l-x)=-f(t, l+x), \\
g(t,-x)=-g(t, x), & g(t, l-x)=-g(t, l+x),
\end{array}
$$

The functions $u_{0}(x), v_{0}(x), f(t, x), g(t, x)$ continued in this way are used as the input data for the Cauchy problem

$$
\left\{\begin{array}{l}
u_{t}(t, x)+a_{11}(t) u(t, x)+a_{12}(t) v(t, x)=\mu_{1} u_{x x}(t, x)+v(t, x) u_{x}(t, x)+r(t) f(t, x), \\
\varepsilon v_{t}(t, x)+a_{21}(t) u(t, x)+a_{22}(t) v(t, x)=\mu_{2} v_{x x}(t, x)+u(t, x) v_{x}(t, x)+g(t, x),
\end{array}\right.
$$

$\varepsilon$ is const, $\varepsilon \in(0,1]$,

$$
\begin{array}{ll}
u(0, x)=u_{0}(x), & x \in(-\infty,+\infty), \\
v(0, x)=v_{0}(x), & x \in(-\infty,+\infty) .
\end{array}
$$

Split the problem (32)-(34):

$$
\left\{\begin{array}{l}
u_{t}^{\tau}(t, x)=4 \mu_{1} u_{x x}^{\tau}(t, x), \\
\varepsilon v_{t}^{\tau}(t, x)=4 \mu_{2} v_{x x}^{\tau}(t, x), \quad j \tau<t \leqslant\left(j+\frac{1}{4}\right) \tau,
\end{array}\right.
$$




$$
\begin{gathered}
\left\{\begin{array}{l}
u_{t}^{\tau}(t, x)+4 a_{11}(t) u^{\tau}(t, x)=0, \\
\varepsilon v_{t}^{\tau}(t, x)+4 a_{22}(t) v^{\tau}(t, x)=0,
\end{array} \quad\left(j+\frac{1}{4}\right) \tau<t \leqslant\left(j+\frac{1}{2}\right) \tau,\right. \\
\left\{\begin{array}{l}
u_{t}^{\tau}(t, x)=4 v^{\tau}\left(t-\frac{\tau}{4}, x\right) u_{x}^{\tau}(t, x), \\
\varepsilon v_{t}^{\tau}(t, x)=4 u^{\tau}\left(t-\frac{\tau}{4}, x\right) v_{x}^{\tau}(t, x), \quad\left(j+\frac{1}{2}\right) \tau<t \leqslant\left(j+\frac{3}{4}\right) \tau
\end{array}\right. \\
\left\{\begin{array}{r}
u_{t}^{\tau}(t, x)+4 a_{12}(t) v^{\tau}\left(t-\frac{\tau}{4}, x\right)= \\
=4 \frac{\psi(t)+a_{12}(t) v^{\tau}\left(t-\frac{\tau}{4}, x^{0}\right)-\mu_{1} u_{x x}^{\tau}\left(t-\frac{\tau}{4}, x^{0}\right)-v^{\tau}\left(t-\frac{\tau}{4}, x^{0}\right) u_{x}^{\tau}\left(t-\frac{\tau}{4}, x^{0}\right)}{f\left(t, x^{0}\right)} f(t, x), \\
\varepsilon v_{t}^{\tau}(t, x)+4 a_{21}(t) u^{\tau}\left(t-\frac{\tau}{4}, x\right)=4 g(t, x), \quad\left(j+\frac{3}{4}\right) \tau<t \leqslant(j+1) \tau, \\
u^{\tau}(0, x)=u_{0}(x), \\
v^{\tau}(0, x)=v_{0}(x) .
\end{array}\right.
\end{gathered}
$$

Let $u^{\tau}(t, x), v^{\tau}(t, x)$ be a solution to the split problem. Let us show that $u^{\tau}(t, x), v^{\tau}(t, x)$ satisfy the conditions

$$
\begin{aligned}
& u^{\tau}(t,-x)=-u^{\tau}(t, x), \quad u^{\tau}(t, l-x)=-u^{\tau}(t, l+x), \\
& v^{\tau}(t,-x)=-v^{\tau}(t, x), \quad v^{\tau}(t, l-x)=-v^{\tau}(t, l+x) .
\end{aligned}
$$

At the first fractional step, using the integral representation, we obtain

$$
\begin{aligned}
& u^{\tau}(t, x)=\int_{-\infty}^{+\infty} u_{0}(\xi) \frac{1}{4 \sqrt{\pi t \mu_{1}}} e^{-\frac{(x-\xi)^{2}}{12 \mu_{1} t}} d \xi \\
& v^{\tau}(t, x)=\int_{-\infty}^{+\infty} v_{0}(\xi) \frac{1}{4 \sqrt{\pi t \mu_{2}}} e^{-\frac{(x-\xi)^{2}}{12 \mu_{2} t}} d \xi
\end{aligned}
$$

Let us check the first conditions from (41) and (42)

$$
\begin{aligned}
& u^{\tau}(t,-x)+u^{\tau}(t, x)=\int_{-\infty}^{+\infty} u_{0}(\xi) \frac{1}{4 \sqrt{\pi t \mu_{1}}}\left(e^{-\frac{(x-\xi)^{2}}{12 \mu_{1} t}}+e^{-\frac{(x+\xi)^{2}}{12 \mu_{1} t}}\right) d \xi . \\
& v^{\tau}(t,-x)+v^{\tau}(t, x)=\int_{-\infty}^{+\infty} v_{0}(\xi) \frac{1}{4 \sqrt{\pi t \mu_{2}}}\left(e^{-\frac{(x-\xi)^{2}}{12 \mu_{2} t}}+e^{-\frac{(x+\xi)^{2}}{12 \mu_{2} t}}\right) d \xi .
\end{aligned}
$$

The integrand changes sign when $\xi$ is replaced by $-\xi$, therefore, the integrals are equal to 0 . The second conditions from (41) and (42) are verified similarly by replacing $\eta=l-\xi$ the variable of integration.

At the second fractional step, $u^{\tau}(t, x), v^{\tau}(t, x)$ have the form

$$
\begin{aligned}
& u^{\tau}(t, x)=u^{\tau}\left(\frac{\tau}{4}, x\right) e^{4 \int_{\tau}^{t} a_{11}(\eta) d \eta}, \quad \frac{\tau}{2}<t \leqslant \frac{\tau}{4}, \\
& v^{\tau}(t, x)=v^{\tau}\left(\frac{\tau}{4}, x\right) e^{4 \int_{\tau}^{t} a_{22}(\eta) d \eta}, \quad \frac{\tau}{2}<t \leqslant \frac{\tau}{4} .
\end{aligned}
$$


Consequently,

$$
\begin{aligned}
& u^{\tau}(t,-x)+u^{\tau}(t, x)=\left(u^{\tau}\left(\frac{\tau}{4},-x\right)+u^{\tau}\left(\frac{\tau}{4}, x\right)\right) e^{4 \int_{\frac{\tau}{4}}^{t} a_{11}(\eta) d \eta}, \quad \frac{\tau}{2}<t \leqslant \frac{\tau}{4}, \\
& v^{\tau}(t,-x)+v^{\tau}(t, x)=\left(v^{\tau}\left(\frac{\tau}{4},-x\right)+v^{\tau}\left(\frac{\tau}{4}, x\right)\right) e^{4 \int_{\frac{\tau}{4}}^{t} a_{22}(\eta) d \eta}, \quad \frac{\tau}{2}<t \leqslant \frac{\tau}{4} .
\end{aligned}
$$

The conditions (41) and (42) follows from the first fractional step.

At the third fractional step, we use Lemma 1.

Lemma 1. Let the function $u(t, x)$ be a solution to the equation $u_{t}=a(t, x) u_{x}$ in the domain $D=\left\{(t, x) \mid t_{0}<t<t_{1}, x \in \Re\right\}$ with the initial condition $u\left(t_{0}, x\right)=u_{0}(x)$. Let the function $(a, t, x)$ satisfy the Lipschitz condition in $x$ and the relations

$$
a(t, c+x)=-a(t, c-x), \quad u_{0}(c+x)=u_{0}(c-x), \quad c \text { is a const }
$$

hold. Then the function $u(t, x)$ satisfies the relation $u(t, c+x)=-u(t, c-x)$.

The proof of Lemma 1 is presented in [8].

Where do we get the fulfillment of the conditions (41) and (42).

At the fourth fractional step, we get

$$
\begin{gathered}
u^{\tau}(t, x)=u^{\tau}\left(\frac{3 \tau}{4}, x\right)+4 \int_{\frac{3 \tau}{4}}^{t}\left(a_{12}(\eta) v^{\tau}\left(\eta-\frac{\tau}{4}, x\right)+\right. \\
\left.+\frac{\psi(\eta)+a_{12}(t) v^{\tau}\left(\eta-\frac{\tau}{4}, x^{0}\right)-\mu_{1} u_{x x}^{\tau}\left(\eta-\frac{\tau}{4}, x^{0}\right)-v^{\tau}\left(t-\frac{\tau}{4}, x^{0}\right) u_{x}^{\tau}\left(t-\frac{\tau}{4}, x^{0}\right)}{f\left(\eta, x^{0}\right)} f(\eta, x)\right) d \eta \\
v^{\tau}(t, x)=v^{\tau}\left(\frac{3 \tau}{4}, x\right)+4 \frac{1}{\varepsilon} \int_{\frac{3 \tau}{4}}^{t}\left(a_{21}(\eta) u^{\tau}\left(\eta-\frac{\tau}{4}, x\right)+g(\eta, x)\right) d \eta
\end{gathered}
$$

Let us check the first conditions from (41) and (42)

$$
\begin{aligned}
& u^{\tau}(t,-x)+u^{\tau}(t, x)=u^{\tau}\left(\frac{3 \tau}{4}, x\right)+u^{\tau}\left(\frac{3 \tau}{4},-x\right)+ \\
& +4 \int_{\frac{3 \tau}{4}}^{t}\left(a_{12}(\eta)\left(v^{\tau}\left(\eta-\frac{\tau}{4}, x\right)+v^{\tau}\left(\eta-\frac{\tau}{4},-x\right)\right)+\right. \\
& +\frac{\psi(\eta)+a_{12}(t) v^{\tau}\left(\eta-\frac{\tau}{4}, x^{0}\right)-\mu_{1} u_{x x}^{\tau}\left(\eta-\frac{\tau}{4}, x^{0}\right)-v^{\tau}\left(t-\frac{\tau}{4}, x^{0}\right) u_{x}^{\tau}\left(t-\frac{\tau}{4}, x^{0}\right)}{f\left(\eta, x^{0}\right)} \times \\
& \times(f(\eta, x)+f(\eta,-x))) d \eta=0, \\
& v^{\tau}(t,-x)+v^{\tau}(t, x)=v^{\tau}\left(\frac{3 \tau}{4}, x\right)+v^{\tau}\left(\frac{3 \tau}{4},-x\right)+ \\
& +4 \frac{1}{\varepsilon} \int_{\frac{3 \tau}{4}}^{t}\left(a_{21}(\eta)\left(u^{\tau}\left(\eta-\frac{\tau}{4}, x\right)+u^{\tau}\left(\eta-\frac{\tau}{4},-x\right)\right)+(g(\eta, x)+g(\eta,-x))\right) d \eta=0 .
\end{aligned}
$$

The second conditions from (41) and (42) are obviously also satisfied. 
We have proved that the conditions (41) and (42) are satisfied at the zero integer step. Arguing in the same way at the next steps, we obtain that the conditions (41) and (42) are satisfied for all $t \in\left[0, t^{*}\right]$. Substituting $x=0$ in (41) and (42), we get

$$
\begin{aligned}
& u^{\tau}(t, 0)=u^{\tau}(t, l)=0, \quad t \in\left[0, t^{*}\right] \\
& v^{\tau}(t, 0)=v^{\tau}(t, l)=0, \quad t \in\left[0, t^{*}\right] .
\end{aligned}
$$

Theorem 3.1. Let conditions (28)-(31) and the conditions of Theorem 1 hold. The components $u, v$ of the solution $(u, v, r)$ to problem (1)-(3) are periodic functions in the variable $x$ with period $2 l$ and satisfy

$$
\frac{\partial^{2 m} u(t, 0)}{\partial x^{2 m}}=\frac{\partial^{2 m+1} u(t, l)}{\partial x^{2 m}}=\frac{\partial^{2 m+1} v(t, 0)}{\partial x^{2 m}}=\frac{\partial^{2 m+1} v(t, l)}{\partial x^{2 m}}=0, \quad m=0,1, \ldots, \frac{p+4}{2} .
$$

\section{References}

[1] Yu.Ya.Belov, An Identification Problem of Source Function for One Semievolutionary System, Journal of Siberian Federal University, Series: Mathematics and Physics, 3(2010), no. 4, 487-499.

[2] Yu.Ya.Belov, V.G.Kopylova, Determination of Source Functions in Composite Type System of Equations, Journal of Siberian Federal University, Series: Mathematics and Physics, 72014, no. 3, 275-288.

[3] Yu.Ya.Belov, An Identification Problem of Source Function in the System of Composite type, Journal of Siberian Federal University. Mathematics and Physics, 4(2011), no. 4, 445-457 (in Russian).

[4] Yu.Ya.Belov, V.G.Kopylova, On some identification problem for source function to one semievolutionary system, Journal of Inverse and Ill-posed Problems, 20(2012), no.5-6, 723-743.

[5] N.N.Yanenko, Fractional steps for solving multidimensional problems of mathematical physics, Novosibirsk, Nauka, 1967.

[6] Yu.Ya.Belov, S.A.Cantor, The method of weak approximation, Krasnoyarsk State University, 1990.

[7] L.V.Kantorovich, G.P.Akilov, Function analysis, Moscow, Nauka, 1977.

[8] Yu.Ya.Belov, K.V Korshun, An Identification Problem of Source Function in the Burgerstype Equation, Journal of Siberian Federal University. Mathematics and Physics, 5(2012), no. $4,497-506$.

[9] A.I.Prilepko, D.G.Orlovsky, I.A.Vasin, Methods for Solving Inverse Problems in Mathematical Physics, New York, Marcel Dekkar, Inc., 1999.

[10] V.G.Romanov, Inverse problems for differential equations, Novosibirsk, NGU, 1978. 


\title{
О разрешимости задачи идентификации функции источника в квазилинейной параболической системе уравнений в ограниченных и неограниченных областях
}

\section{Вера Г. Копылова}

Игорь В. Фроленков

Сибирский федеральный университет Красноярск, Российская Федерация

\begin{abstract}
Аннотация. В работе рассматривается задача идентификации функции источника в одном из двух уравнений квазилинейной системы двух параболических уравнений. Рассматривается случай данных Коши в неограниченной области, а также случай краевых условий первого рода в прямоугольной области. Изучен вопрос существования и единственности решения. Для доказательства используется метод расщепления на дифференциальном уровне, известный как метод слабой аппроксимации. Решение получено на малом временном интервале в классе достаточно гладких ограниченных функций.
\end{abstract}

Ключевые слова: обратная задача, система квазилинейных уравнений, определение функции источника, метод слабой аппроксимации, малый параметр. 